\title{
3D Morphometry SEM Analysis of Microvascular Networks and Calculation of Vessel Angioarchitecture Optimalities
}

\author{
Bernd Minnich $^{1}$, Sebastian Margiol ${ }^{2}$, Josef Frysak ${ }^{2}$ and Edward Bernroider ${ }^{2}$ \\ ${ }^{1 .}$ University of Salzburg, Dept. Cellbiology \& Physiology, Div. Animal Structure \& Function, Vascular \\ and Exercise Biology Unit, Salzburg, Austria. \\ 2. Vienna University of Economics and Business, Inst. Information Management and Control, Vienna, \\ Austria.
}

Scanning electron microscopes (SEMs) are potential tools for morphological research. They have a great resolution and a high depth of focus, which gives SEM micrographs pseudo three-dimensionality. Adversely, the high depth of focus prevents accurate dimensional or spatial measurements of imaged microstructures from either the SEM video-display, printed micrographs or from photo-negatives. Macroscopic objects are viewed close up using binocular vision. Binocular vision is also used in microscopy where stereophotogrammetry and related techniques applying stereo paired images, and a variety of hardware tools calculate the third dimension (z-coordinate) using the parallax.

A method (3D-morphometry, ComServ, Austria) for dimensional and angular measurements of microstructures imaged in the SEM was first developed in our lab in 1999 [1]. It uses digitized stereo paired images frame-grabbed (slow scan) directly from the SEM's photo-display, vector equation-based algorithms for the calculation of spatial coordinates and derived distance- as well as angular measurements. It offers dynamic data exchange to open source software together with online graphs of frequency distributions of measured variables. Formulas for central perspective depth computation allow the overall error to be less than 1.0\%. Meanwhile (2012), the method was further improved and the new java based software M3 is suitable to be used with Windows 7 and 8 operating systems. A modern graphical user interface (GUI) (Fig.1), a new data interface (open office) is supported and an improved usability facilitates measurement processes [2].

This technique is currently used to analyse (a) the geometry of microvascular trees in terms of vascular parameters (i.e. diameters, interbranching distances, branching angles and intervascular distances) and (b) to determine bifurcation indices and area ratios followed by a calculation of optimality principles which are given in arterial bifurcations respectively venous mergings. Moreover the program now allows to generate anaglyphic 3D images (Fig.2) and to calculate optimality principles underlying the construction and maintenance of such delicate vascular networks (i.e. principle of minimal lumen volume, minimal pumping power, minimal lumen surface and minimal endothelial shear force) [3-4] (Fig.3). In our current research we use M3 to examine developmental and regression processes of the microvasculature of different organs in various animals such as the South African clawed toad (Xenopus laevis Daudin) [5-7] and also to explore the angioarchitecture of the human great saphenous vein (Vena saphena magna) which is a critical factor for bypass crafting during coronary surgery in men [8].

\section{References:}

[1] B Minnich et al, J. Microsc., 195 (1999) 23-33.

[2] B Minnich et al in "Current microscopy contributions to advances in science and technology", eds. A Mendes-Vilas and J D Alvarez, Formatex, (2012) 191-199.

[3] M LaBabera, Science, 249 (1990) 992-1000.

[4] CD Murray, J. Gen. Physiol., 9 (1926) 835-841. 
[5] A Lametschwandtner et al, Arch. Histol. \& Cytol., 73(1) (2010) 55-64.

[6] A Lametschwandtner et al, Italian J. Zoology, 78(1) (2011) 27-34.

[7] A Lametschwandtner et al, Anatomical Sciences International, (2012) 87, 88-100.

[8] D Kachlik et al, Central European Journal of Medicine, 3(4) (2008) 475-481.

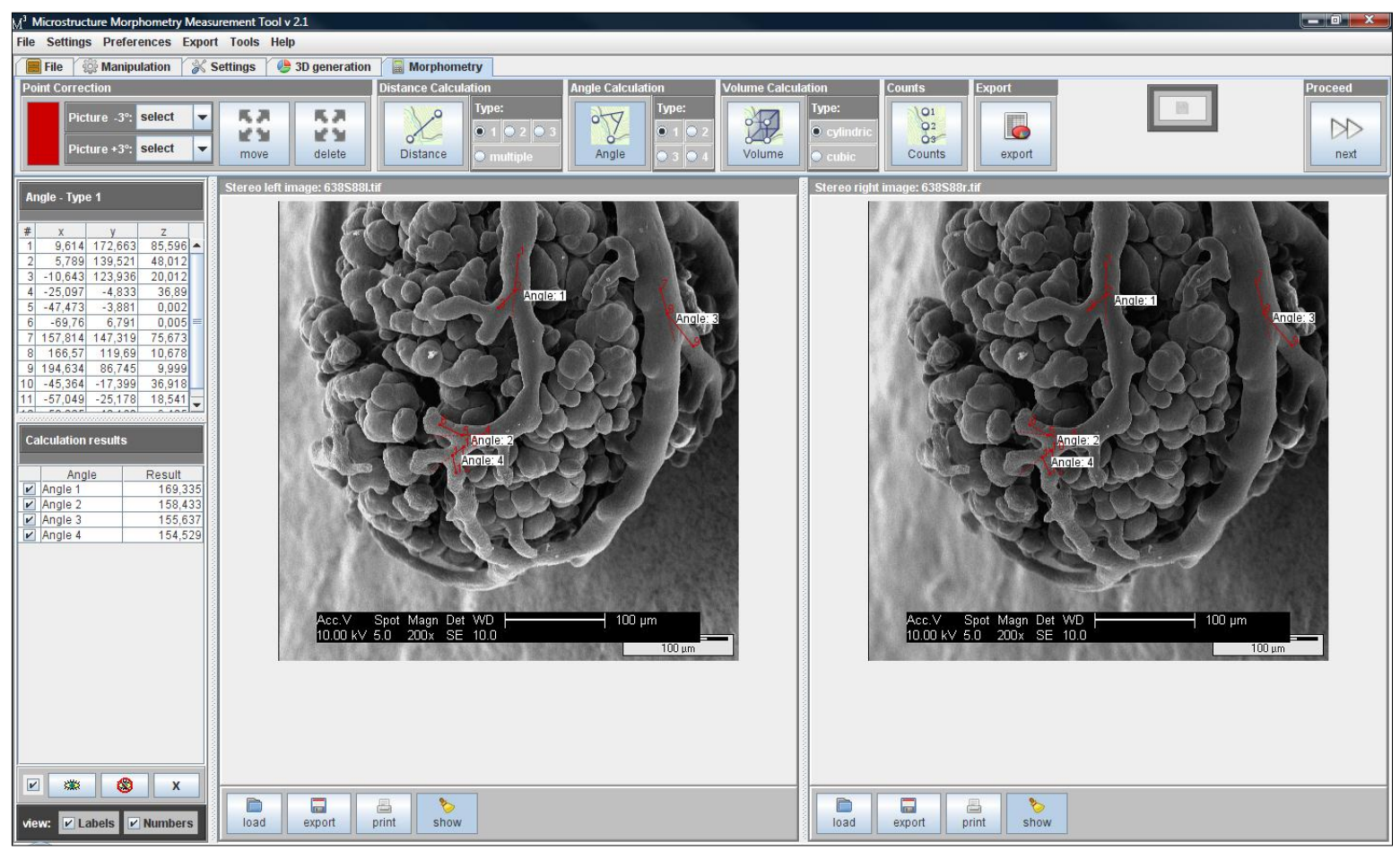

Figure 1. M3 graphical user interface (GUI) showing the morphometry screen while measuring branching angles of feeding arteries of the spleen of the adult Xenopus laevis Daudin.
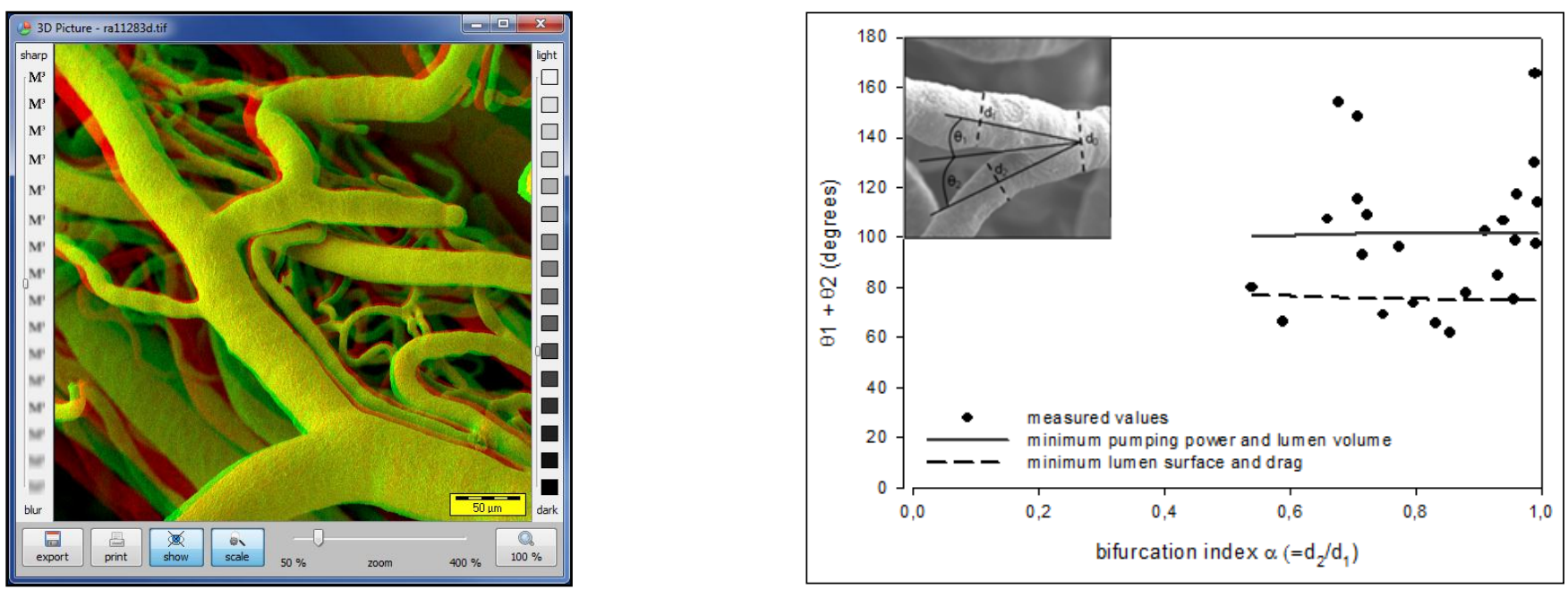

Figure 2. Anaglyphic 3D image representing a vascular corrosion cast of a microvascular network examined under an ESEM (FEI XL-30).

Figure 3. Scatterplot showing an optimality diagram of the brain microvascular bed (rhombencephalon) of the sterlet (Acipenser ruthenus). Vascular bifurcation with indications of vessel diameters and branching angles (insert). 